82 Magma

\title{
Notas SObre Le Club des HaChICHINS, DE THÉOPHILE GAUTIER
}

Bruno Penteado Natividade Moreto*

Resumo

O trabalho tece alguns comentários sobre Le Club des Hachichins, de Téophile Gautier, numa abordagem que releva o caráter persuasivo, no texto literário, da sugestão do consumo do haxixe. O recurso é calculado e resulta na oficialização do relato ficcional (devido à proximidade com o compte-rendu), aproximando-o do âmbito do real, sem que o texto perca, no entanto, seus traços fantásticos.

Abstract

This paper focuses on Gautier's Le Club des Hachichins with regard to the persuasive role hashish plays in the text. However, the intertwinement of fiction and reality caused by the presence of the drug does not annihilate the irruption of fantastic elements in the story.
Palavras-chave

Literatura

Francesa;

Théophile

Gautier;

Tópicas do

século XIX.

Keywords

French

Literature;

Théophile

Gautier;

XIXth-century

leitmotivs.

* Mestrando em Teoria Literária e Literatura Comparada (FFLCH-USP). 


\section{O Imaginário do haxixe}

$P$

or volta dos anos 1840, a literatura francesa ligou-se intimamente ao que chamaremos imaginário do haxixe: ela assimilou aspectos do discurso médico, que tentava estabelecer os efeitos que o haxixe produzia no corpo humano; ${ }^{1}$ do discurso místico, que tentava definir os efeitos do haxixe na alma; ${ }^{2}$ e, na tradicional fascinação pelo Oriente, encontrou os pilares para essa tópica que começava a se desenvolver. $^{3}$

1 "J'avais vu dans le hachisch, ou plutôt dans son action sur les facultés morales, un moyen puissant, unique, d'exploration en matière de pathogénie mentale; je m'étais persuadé que par elle on devait pouvoir être initié aux mystères de l'aliénation, remonter à la source cachée de ces désordres si nombreux, si variés, si étranges qu'on a l'habitude de désigner sous le nom collectif de folie". Moreau de Tours, Jacques-Joseph. Du Hachisch et de l'Aliénation Mentale (1845). Paris/Genève: Slatkine Reprints, 1980, p. 29-30. Collection Ressources. Depois de afirmar que "le haschisch est inutile et dangereux", Charles Baudelaire deixa entrever, em uma nota, seu desprezo por Moreau de Tours: "Il ne faut mentionner que pour mémoire la tentative faite récemment pour appliquer le haschisch à la cure de la folie. Le fou qui prend du haschisch contracte une folie qui chasse l'autre, et quand l'ivresse est passée, la vraie folie, qui est l'état normal du fou, reprend son empire, comme chez nous la raison et la santé. Quelqu'un s'est donné la peine d'écrire un livre là-dessus. Le médecin qui a inventé ce beau système n'est pas le moins du monde philosophe". BAUDELAIRE, Charles. Du vin et du haschisch comparés comme moyens de multiplication de l'individualité. Les Paradis Artificiels. Chronologie et introduction par Marcel A. Ruff. Paris: Garnier-Flammarion, 1966, p. 187.

2 "J'ai la conviction que le haschich développe chez nous l'état spirituel dans lequel chacun peut trouver des solutions répondant à ses affections. Je sais que beaucoup d'étudiants à Paris en prennent des doses raisonnables pour développer chez eux des solutions nécessaires à leurs études, et qu'on retire toujours quelque chose d'instructif de l'état dans lequel il nous met". CAHAGNET, Louis Alphonse. Sanctuaire du Spiritualisme; Etude de l'âme humaine d'après le somnambulisme et l'extase. Paris: LAC, 1850, p.115-16.

3 "Il existait jadis en Orient un ordre de sectaires redoutables commandé par un cheik qui prenait le titre de Vieux de la Montagne, ou prince des Assassins. Ce Vieux de la Montagne était obéi sans réplique [...]. Par quels artifices le Vieux de la Montagne obtenait-il une abnégation si complète? Au moyen d'une drogue merveilleuse dont il possédait le recette, et qui a la propriété de 
Mas por que "imaginário do haxixe"? Milner responde à questão com justeza: "Entendons par là [o imaginário das drogas] l'ensemble des représentations, images, symboles, élaborations mythiques par lesquels les individus ou les groupes réagissent aux réalités auxquelles ils sont confrontés". ${ }^{4}$ Os efeitos reais do haxixe e suas relações com a criação literária interessam enquanto fontes de uma tópica, de um lugar-comum observável em inúmeros escritores franceses do mesmo momento histórico. Milner complementa: "Le XIX ${ }^{e}$ siècle a favorisé l'usage chez ceux-ci [os europeus] en développant les échanges avec les pays exotiques, en intensifiant leur action par des moyens chimiques [...]". ${ }^{5}$ É efetivamente interessante notar que:

Le succès rencontré par ces drogues résulte cependant de facteurs moins matériels. Comme les instruments d'optique auxquels je faisais allusion [em sua obra La Fantasmagorie. Essai sur l'optique fantastique], elles se révèlent aptes à induire dans la conscience des modifications répondant aux besoins créés par l'évolution de la culture, et elles introduisent à leur tour dans cette même culture des comportements, des représentations et des idéaux qui n'existaient que de manière très sporadique aux époques precedentes. ${ }^{6}$

procurer des hallucinations éblouissantes[... - c'est à dire du hachich, d'où vient hachichin, mangeur de hachich, racine du mot assassin, dont l'acception féroce s'explique parfaitement par les habitudes sanguinaires des affidés du Vieux de la Montagne". GAUTIER, Théophile. Le Club des Hachichins. Récits Fantastiques. Chronologie, introduction et notes par Marc Eigeldinger. Paris: Flammarion, 1981, p. 215-16. "M. Sylvestre de Sacy a démontré, en s'appuyant sur différents textes arabes, que le mot assassin était la corruption du mot hachischin, et qu'il avait été donné aux Ismaéliens parce qu'ils faisaient usage d'une liqueur enivrante appelée hachisch. [...] Ceux qui se livrent à cet usage sont encore aujourd'hui appelés Hachischins ou Hachaschins; et ces deux expressions font voir pourquoi les Ismaéliens ont été nommés par les historiens latins des croisades tantôt assissini, tantôt assassini". MOREAU DE TOURS, op. cit., 1980, p. 11-2. "On ne peut douter que les écrivains des croisades ne les aient confondus souvent avec les Ismaéliens, dont une secte a été cette fameuse association des Assassins qui fut un instant la terreur de tous les souverains du monde; mais ces derniers occupaient le Curdistan, et leur cheik-el-djebel, ou Vieux de la Montagne, n'a aucun rapport avec le prince de la montagne du Liban". NERVAL, Gérard de. Voyage en Orient. vol. 2. Chronologie, introduction, bibliographie, lexique et notes par Michel Jeanneret. Paris: Flammarion, 1980 , p. 57, v. 2. "Les récits de Marco Polo dont on s'est à tort moqué, comme de quelques autres voyageurs anciens, ont été vérifiés par les savants et méritent notre créance. Je ne raconterai pas après lui comment le Vieux de la Montagne enfermait, après les avoir énivrés de haschisch (d'où Haschischins ou Assassins), dans un jardin plein de délices, ceux de ses plus jeunes disciples à qui il voulait donner une idée du paradis, récompense entrevue, pour ainsi dire, d'une obéissance passive et irréfléchie. Le lecteur peut, relativement à la société secrète des Haschischins, consulter le livre de M. De Hammer et le mémoire de M. Silvestre de Sacy, contenu dans le tome XVI des Mémoires de l'Académie des Inscriptions et Belles-Lettres, et, relativement à l'étymologie du mot assassin, sa lettre au rédacteur du Moniteur, insérée dans le numéro 259 de l'année 1809. [...] Le haschisch, en effet, nous vient de l'Orient; les propriétés excitantes du chanvre étaient bien connues dans l'ancienne Egypte, et l'usage en est très-répandu, sous différents noms, dans l'Inde, dans l'Algérie et dans l'Arabie Heureuse". BAUDELAIRE, Charles. Le Poème du Haschisch, op. cit., 1966, p. 31.

${ }^{4}$ MILNER, Max. L'Imaginaire des Drogues. De Thomas de Quincey à Henri Michaux. Paris: Gallimard, 2000 , p. 7.

${ }^{5}$ Ibid., p. 8.

${ }^{6}$ Ibid. 
Nossa proposta é tentar demonstrar, em relação ao conto Le Club des Hachichins, de Théophile Gautier, que o consumo do haxixe pelo escritor, ainda que possa ser "real", funciona, no texto, como um instrumento retórico que, por sua vez, contribui para a criação de uma atmosfera artificial. A aparência de compte-rendu do texto, longe de reforçar os laços entre experiência vivida e criação artística, aliada a tal retórica do uso do haxixe, produz um terceiro efeito, que poderíamos chamar "efeito de simulacro". ${ }^{7}$

Milner menciona as "soirées" organizadas por Moreau de Tours, que havia conhecido o haxixe ao longo de uma série de viagens ao Egito e ao Oriente Médio. Ao retornar à França, passou a oferecer a possibilidade de experimentar a droga a diversos indivíduos curiosos, dentre os quais Gautier, ${ }^{8}$ cuja primeira experiência foi relatada em La Presse em forma de compte-rendu (Le Hachich) em 19 de julho de 1843. Milner ressalta que "Moreau lui-même reconnaît qu'il y a là 'peut-être (...) un peu d'exagération dans la forme', mais, tout heureux d'avoir à faire à un si brillant interprète, il certifie 'la véracité de l'écrivain, qui, en définitive, ne fait qu'exprimer des sensations familières à ceux qui ont quelque expérience du hachisch". ${ }^{9}$

Rigoli nota que Moreau de Tours, "dès les premiers pages du traité", reproduz "presque intégralement son article de 1843 sur 'Le Hachich". ${ }^{10}$ O que foi ocultado, além de algumas informações técnicas sobre o haxixe, às quais o alienista dedica considerável parte de seu livro, é justamente o início do relato, no qual Gautier afirma que

Le butin théâtral a été, comme vous le pouvez voir, bien mince cette semaine [...] Manquant de spectacles, nous avons décidé de nous en donner un à nous-mêmes sans sortir de notre chambre et dans le coin de notre sofa [...] Quelques amis orientalistes nous avaient promis plusieurs fois de nous en faire goûter; mais, soit difficulté de se procurer la précieuse pâte, soit toute autre raison, le projet n'avait pas encore été réalisé. Il l'a été enfin hier, et l'analyse de nos sensations remplacera le compte-rendu des pièces qu'on n'a pas jouées. ${ }^{11}$

Não havendo peças teatrais "aceitáveis" sendo encenadas, a única saída é encenar o espetáculo do haxixe; Gautier aproxima deliberadamente as sensações experimentadas por conta do uso da droga ao compte-rendu de peças não encenadas. A teatralização da experiência do haxixe, ou seja, a ficcionalização de uma experiência central na obra de Moreau de Tours, era compreensivelmente pouco oportuna ao alienista. Além do mais, o ato de censura, o silenciamento da ficcionalização de uma experiência sublinha as suspeitas sobre sua "incontestable véra-

7 Lembremos Barthes e o efeito de real.

8 MILNER, op. cit., 2000, p. 69-70.

9 MILNER, op. cit., 2000, p. 72.

${ }^{10}$ RIGOLI, Juan. Lire le Délire. Aliénisme, rhétorique et littérature en France au XIXe siècle. Paris: Fayard, 2001, p. 443.

${ }^{11}$ GAUTIER, Théophile. Le Hachich. Annales Médico-Psychologiques, Tome II, novembre 1843, p. 490. 
cité". A verossimilhança do relato (algo como "tudo se deu como descrito pois o haxixe abre as portas para outros estados mentais") é persuasiva e, por sua vez, permite a ele ser fantástico. ${ }^{12} \mathrm{O}$ haxixe faz acreditar que o caráter artificial e fantástico do texto é verdadeiro enquanto experiência vivida. É evidente que Gautier fará bom uso dessa retórica sub-reptícia. ${ }^{13}$

Algum tempo depois, Gautier publica Le Club des Hachichins, que supostamente dá conta de uma das noites de "fantasia" organizadas por Moreau de Tours no Hotel Pimodan. As especulações sobre o grau de veracidade dos fatos narrados no conto, especialmente devido ao texto publicado em La Presse, não tardaram a aparecer. Aliás, Gautier, num estudo que fez sobre Baudelaire, afirma que, à matéria ficcional, ele acrescentou suas "propres hallucinations." No entanto, como não existe um instrumento que possa medir o papel da experiência do haxixe na criação do conto, resta-nos o contentamento da função indutora da droga, obviamente no sentido retórico. Ou seja, a simples menção ao sema "haxixe" abre possibilidades de persuasão e facilita o acesso ao imaginário fantástico da época.

\section{O haxixe enquanto sema retórico}

O primeiro período do conto Le Club des Hachichins causa de imediato uma sensação de inquietante estranheza:

Un soir de décembre, obéissant à une convocation mystérieuse, rédigée en termes énigmatiques compris des affiliés, inintelligibles pour d'autres, j'arrivai dans un quartier lointain, espèce d'oasis de solitude au milieu de Paris, que le fleuve, en l'entourant de ses deux bras, semble défendre contre les empiétements de la civilisation, car c'était dans une vieille maison de l'île Saint-Louis, l'hôtel Pimodan, bâti par Lauzun, que le club bizarre dont je faisais partie depuis peu tenait ses séances mensuelles, où j’allais assister pour la première fois. ${ }^{14}$

${ }^{12}$ Para precisar o termo empregado, Todorov propõe que o fantástico literário se caracteriza principalmente pela sensação de incerteza provocada no leitor: "Lambigüité se maintient jusqu'à la fin de l'aventure: réalité ou rêve? vérité ou illusion? Ainsi se trouve-t-on amené au cœur du fantastique. Dans un monde qui est bien le nôtre, celui que nous connaissons, sans diables, sylphides, ni vampires, se produit un événement qui ne peut s'expliquer par les lois de ce même monde familier. Celui qui perçoit l'événement doit opter pour l'une des deux solutions possibles: ou bien il s'agit d'une illusion des sens, d'un produit de l'imagination et les lois du monde restent alors ce qu'elles sont; ou bien l'événement a véritablement eu lieu, il est partie intégrante de la réalité, mais alors cette réalité est régie par des lois inconnues de nous. [...] Le fantastique occupe le temps de cette incertitude; dès qu'on choisit l'une ou l'autre réponse, on quitte le fantastique pour entrer dans un genre voisin, l'étrange ou le merveilleux. Le fantastique, c'est l'hésitation éprouvée par un être qui ne connaît que les lois naturelles, face à un événement en apparence surnaturel. Le concept de fantastique se définit donc par rapport à ceux de réel et d'imaginaire". TODOROV, Tzvetan. Introduction à la Littérature Fantastique. Paris: Seuil, 1970, p. 29.

${ }^{13}$ Sobre o relato de Gautier, Milner escreve que "la circonstance est évidemment inventée, car nous savons que le docteur Moreau était présent, et il apparaît, d'après le contexte, que Gautier n'était pas seul à consommer la drogue. Mais la transformation de l'expérience en spectacle - ce que Baudelaire appellera plus tard le 'théâtre de Séraphin' - n'en est que plus frappante". MILNER, op. cit., p. 72 .

${ }^{14}$ GAUTIER, op. cit., 1981, p. 211-12. 
Há, na verdade, dois "iniciados": o narrador, que provará o haxixe pela primeira vez, e o leitor, que ainda não sabe o que está havendo. O período longo apenas contribui para tal sensação de incerteza: a falta de pausas trunca o tempo narrativo, e o excesso de informações para um primeiro parágrafo agrava o transtorno.

$\mathrm{O}$ percurso feito ao longo do primeiro capítulo insiste em tal procedimento. Depois de sua chegada ao hotel, o narrador entra em uma sala onde, após tomar uma xícara de café, começa a comer uma geleia esverdeada, embora, como afirma, ainda não tenha jantado. Gautier está ciente de sua escritura pouco ortodoxa: "Cette inversion dans les habitudes culinaires a sans doute surpris le lecteur; en effet, il n'est guère d'usage de prendre le café avant la soupe, et ce n'est en général qu'au dessert que se mangent les confitures. La chose assurément mérite d'explication". ${ }^{15}$

Tal dispositivo é bastante revelador: ora, um simples relato de uma experiência vivida não precisaria de tal rébus narrativo: não se trata apenas da experiência do consumo do haxixe, mas principalmente da ritualização do discurso.

O que, no entanto, devolve à narrativa seu aspecto de compte-rendu é o segundo capítulo, Parenthèse, no qual o narrador explica a origem do haxixe, como se prepara a confiture verte, bem como explicita que "la pâte verte dont le docteur venait de nous faire une distribution était précisément la même que le Vieux de la Montagne ingérait jadis à ses fanatiques". ${ }^{16}$ A solução do mistério do primeiro capítulo rouba do texto seu caráter inquietante ao dar-lhe ares de experiência relatada. É essencial salientar que a sensação de que "ele viveu o que nos conta" se produz somente após o capítulo explicativo, no qual se introduz o sema "haxixe".

O leitor se pergunta: mas que droga o narrador consumiu? Que tipo de alucinação ele teve? O imaginário do haxixe - e que não se esqueça de que Gautier já havia publicado seu compte-rendu na Presse - começa a produzir resultados: tudo o que se leu e se escutou sobre o haxixe revém. ${ }^{17}$ A estrutura do texto é bastante funcional: Gautier nos coloca em certa disposição passional; primeiramente, ele esconde os "fatos", depois confunde seu leitor e ri-se dele. Apenas mais tarde é que a revelação da chave de leitura do conto - o haxixe - aparece, acompanhada do prenúncio de acontecimentos fantásticos. Nesse sentido, a simples menção do haxixe permite ao discurso ser fantástico sem que fique desprovido de verossimilhança. É preciso relembrar Aristóteles: não se trata do verdadeiro, mas do verossímil.

${ }^{15}$ GAUTIER, op. cit., 1981, p. 214.

${ }^{16}$ GAUTIER, op. cit., 1981, p. 216.

17 "Vous avez entendu parler vaguement des merveilleux effets du haschisch; votre imagination a préconçu une idée particulière, quelque chose comme un idéal d'ivresse; il vous tarde de savoir si la réalité sera décidément à la hauteur de votre esperance". "Vous avez entendu parler vaguement des merveilleux effets du haschisch; votre imagination a préconçu une idée particulière, quelque chose comme un idéal d'ivresse; il vous tarde de savoir si la réalité sera décidément à la hauteur de votre esperance". BAUDELAIRE, op. cit., p. 38. 
Os capítulos seguintes seguem à risca os diferentes estágios da experiência do haxixe, encontrados em diferentes relatos da época. ${ }^{18}$ Agape (a refeição, depois do consumo do haxixe) ${ }^{19}$ Un Monsieur qui n'était pas invité (o início da alucinação, surgimento da personagem Daucus Carota, de um conto de Hoffmann); ${ }^{20}$ Fantasia (o auge da alucinação); ${ }^{21}$ Kief (estágio que sucede a fantasia);22 Le Kief tourne au cauchemar (depois da paz, os efeitos do haxixe revêm brutalmente); ${ }^{23}$ Tread-mill

18 "Les premiers effets sont un plaisir extrême qu'on trouve à s'étendre sur un divan, à fumer et à prendre du café, un dégoût pour toute espèce de mouvement, puis les paupières se contractent comme si la lumière vous faisait mal. Les idées les plus bizarres commencent à surgir; viennent les gros rires, les extravagances soit en paroles soit en action; les Arabes appellent ces derniers effets Fantasia. Au milieu de tout cela la faim, mais une faim presque canine, le vin répugne. A table les effets vont en augmentant, cessent peu à peu, et tout se termine par un sommeil doux et rempli de songes agréables". AUBERT, L. Sur le hachisch et son emploi dans le traitement de la peste. De la Peste ou Typhus d'Orient. Documents et observations recueillis pendant les années 1834 à 1838, en Egypte, en Arabie, sur la Mer Rouge, en Abissynie, à Smyrne et à Constantinople. Paris: Librairie des Sciences Médicales, 1840, p. 213-14. Jacques-Joseph Moreau de Tours, por sua vez, cita os principais fenômenos experimentados, segundo sua ordem de aparição: a) sentimento de felicidade; b) excitação, dissociação das ideias; c) confusão sobre tempo e espaço; d) desenvolvimento da sensibilidade auditiva, influência da música; e) ideias fixas, convicções delirantes; f) lesão das afeições; g) impulsos irresistíveis; h) ilusões, alucinações.

19 "Il faut prendre le hachisch à jeun [...] Le café paraît aider à leur développement". Moreau de Tours, Jacques-Joseph, op. cit., 1980, p. 9. "Ainsi, c'est dit: vous avez même, pour lui donner plus de force et d'expansion, délayé votre dose d'extrait gras dans une tasse de café noir; vous avez pris soin d'avoir estomac libre, reculant vers neuf ou dix heures du soir le repas substantiel, pour livrer au poison toute liberté d'action". Eis o motivo de primeiramente se tomar café, e apenas depois se passar à refeição. BAUDELAIRE, op. cit., 1966, p. 37.

20 "La présence de ce fantastique traditionnel se manifeste avec la plus grande évidence par l'intervention, tout au long des visions provoquées par le hachisch, d'un personnage d'Hoffmann: le nain Daucus Carota de La Fiancée du roi. Celui-ci sera le véritable meneur de jeu du défilé carnavalesque dans lequel le haschischin novice se trouvera entraîné. Les réminiscences culturelles ont, là aussi, une bonne part: personnages de la comédie italienne ou de la pantomime française, êtres composites directement inspirés, comme dans le texte précédent, des tableaux de Bosch ou de Brueghel". (grifo nosso) MILNER, op. cit., 2000, p. 75.

21 "Mais ce n'est qu'avec une dose beaucoup plus élevée qu'on obtiendra les effets que l'on désigne généralement dans le Levant sous la dénomination italienne de fantasia". MOREAU DE TOURS, op. cit., 1980, p. 9.

22 "La troisième phase, séparée de la seconde par un redoublement de crise, une ivresse vertigineuse suivie d'un nouveau malaise, est quelque chose d'indescriptible. C'est ce que les Orientaux appellent le kief; c'est le bonheur absolu. Ce n'est plus quelque chose de toubillonant et de tumultueux. C'est une béatitude calme et immobile. Tous les problèmes philosophiques sont résolus. Toutes les questions ardues contre lesquelles s'escriment les théologiens, et qui font le désespoir de l'humanité raisonnante, sont limpides et claires. Toute contradiction est devenue unité. L'homme est passé dieu". BAUDELAIRE, Du Vin et du Haschisch, comparés comme moyens de multiplication de l'individualité, op. cit., 1966, p 183.

23 "Ces phénomènes, d'assez courte durée d'abord, revinrent comme par bouffées plus longues, plus intenses, plus rapprochées; les intermissions, au contraire, allèrent en s'effaçant”. RECH, M. Des effets du hachisch sur l'homme jouissant de sa raison et sur l'aliéné. In: Annales Médico-Psychologiques. Journal de l'Anatomie, de la Physiologie et de la Pathologie du Système Nerveux. Pathologie. Maladies Mentales, sans references, p. 20. 
(o suplício que sucede o êxtase) ${ }^{24}$ e Ne croyez pas aux chronomètres (sob o efeito do haxixe, a percepção do tempo é peculiar). ${ }^{25}$ Tal ordem apenas reforça a aparência de compte-rendu do conto. Todavia, ela revela, por sua vez, trabalho intelectual intenso: como dissemos, a dispositio segue exatamente as fases da experiência do haxixe descritas em diversos relatos médicos. Não é arbitrariamente que Moreau de Tours, ao escrever sobre o compte-rendu de Gautier que saíra na Presse, afirma que "le hachisch ne pouvait trouver un plus digne interprète que la poétique imagination de M. Gautier; ses effets ne pouvaient être peints avec des couleurs plus brillantes, et j'oserais dire plus locales". ${ }^{26}$ Ainda que o alienista se refira ao compte-rendu e não ao conto, cremos que é justo apropriar-se de seus comentários em relação ao último. Segundo Moreau de Tours, o que faz Gautier é justamente interpretar, produzir mímesis dos efeitos do haxixe num gênero literário. A pintura é "brillante". Há mesmo "des couleurs locales": o afrancesamento de uma experiência oriental se faz explícito.

Portanto, é possível sugerir que o haxixe permite a Gautier fazer com que seu texto tenha ares de compte-rendu, através da utilização de um dos elementos característicos do discurso médico. Além disso, através do mesmo recurso, o caráter fantástico do texto torna-se verossímil, uma vez que os sintomas do consumo de haxixe são, basicamente, o surgimento de alucinações. No primeiro caso, trata-se de trazer ao discurso literário as convenções do discurso médico; no segundo, o "haxixe" (sema) tem considerável apelo retórico.

Enfim, a incerteza de que nos fala Todorov não se inscreve nos fatos narrados no conto: é evidente que se trata de uma sequência de alucinações; está claro que a causa é o haxixe. A incerteza na qual pensamos é de outra ordem. Se o texto tira vantagem do papel persuasivo do haxixe, resta que nos indaguemos qual é o papel criador da droga. Essa dúvida irresolúvel e irresoluta é justamente o grande charme do texto, o que, curiosamente, acaba por inseri-lo no campo do fantástico.

${ }^{24}$ Reproduzimos aqui a nota de Elgeldinger, presente na edição do conto que utilizamos neste trabalho: "tread-mill, moulin à discipline, écureuil au sens ancien du terme, ouvrier ou forçat qui, dans certains métiers artisanaux, faisait tourner une roue destinée au fonctionnement d'un mécanisme ou d'une machine. Après l'extase de la drogue vient le supplice", p. 229.

25 "Le temps semble d'abord se traîner avec une lenteur qui désespère. Les minutes deviennent des heures, les heures des journées [...]; car, si le temps paraît plus long que s'il était mesuré par des horloges terrestres, ce sont les actions ou les faits renfermés dans cet intervalle qui en reculent les limites par l'ampleur". MOREAU DE TOURS, op. cit., 1980, p. 68-9.

${ }^{26}$ MOREAU DE TOURS, op. cit., 1980, p. 20. 